\title{
Implementation of health promoting policies through tailored interventions at health promoting schools and municipalities in Latvia
}

\author{
Inese Gobina ${ }^{1, *}$, Santa Pildava $^{1}$, Elina Millere ${ }^{1}$, Dita Heiberga $^{1}$, Margarita Apine $^{1}$, \\ Ilze Straume ${ }^{2}$, and Aigars Miezitis ${ }^{1}$ \\ ${ }^{1}$ Rìga Stradiņš University, Institute of Public Health, Riga, Latvia \\ ${ }^{2}$ Centre of Disease Prevention and Control, Riga, Latvia
}

\begin{abstract}
Schools and municipalities play an important role in local health promotion. The Institute of Public Health at Rịga Stradinš University in collaboration with the National Centre of Disease Prevention and Control carried out an online survey for studying the experiences and needs of health-promoting schools and municipalities in Latvia in implementing health promotion interventions. The aim of this study was to explore the context for developing new intervention model for a health behaviour change model within the Interreg BaltCityPrevention project "Innovative LifestyleRelated Disease Prevention Model in the Baltic Sea Region." Totally 112 municipalities and 100 schools within the National Health Promoting Networks were targeted. Only schools and municipalities that had previous experiences in health promotion intervention development were included in the analysis. This study showed that school-aged children were the most prevalent target group for the both schools and municipality interventions. The interventions in physical activity and nutrition used to be the most common areas for health behaviour changes. Health promotion schools and municipalities reported several significant differences in experienced difficulties and expected future needs in implementing health promotion interventions, which should be considered for increasing the capacity for better health promotion for both schools and municipalities in Latvia.
\end{abstract}

Key words: health promotion, municipalities, schools.

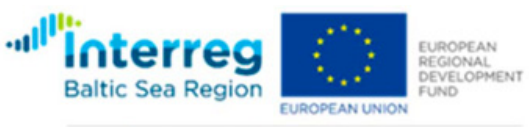

BaltCityPrevention

\section{Introduction}

Health promotion is the key approach for reducing the burden of non-communicable diseases by changing the lifestyles. Creating supportive environments and strengthening community actions have been recognised as important health promotion actions in the World Health Organization (WHO) Ottawa Charter for Health Promotion [1]. Thus, at the local level, schools and municipalities may have a substantial potential in promoting health on both individual and community levels.

\footnotetext{
* Corresponding author: Inese.Gobina@rsu. Iv
}

(C) The Authors, published by EDP Sciences. This is an Open Access article distributed under the terms of the Creative Commons Attribution License 4.0 (http://creativecommons.org/licenses/by/4.0/). 
The existing evidence from systematic reviews suggests that the health promotion approach implemented at schools may contribute to improving child and adolescent health and promotion of healthy lifestyle factors, such as increasing physical activity and vegetable and fruit consumption and reducing tobacco use [2-5]. However, the description of information on interventional elements and the implementation context in the studies is limited, causing difficulties in understanding the interventional development process and understanding the learning experience [6].

Within the World Health Organisation's (WHO) Global School Health Initiative, a HealthPromoting School can be characterised as "a school constantly strengthening its capacity as a healthy setting for living, learning and working” [7]. In Latvia, the existing National Network of Health Promoting Schools was established in 2014, building upon the previous initiatives of Health Promoting Schools' Network started in 1993. The National Network of Health Promoting Schools in Latvia belongs to the larger European Network of Health Promoting Schools, Schools for Health in Europe (https: //www . schoolsforhealth .org).

Studies of determinants of health promotion actions in local contexts are the focus of health promotion research and practice. Health promotion at municipal level involves essential components, such as community organisations, social planning, resource mobilisation, and cross-section collaboration [8-10]. The National Network of Health Promoting Municipalities in Latvia was started in 2013 with the aim of supporting municipalities for community health promotion at the local level by sharing the best practices and increasing the capacity of health promotion practices.

The health behaviour change, aimed at different behavioural domains, is the key instrument in health promotion practices. There are well-developed existing frameworks, including the behaviour change wheel [11], interventional mapping [12], and the PRECEDEPROCEED model [13] for health intervention development. In all of those, several elements such as needs assessment of the target group, understanding the context of health behaviours, identifying determinants of health behaviours, and selecting appropriate tools for behaviour change are recognised as important for developing the interventions of health behaviour change.

This study was implemented within the InterregBaltCityPrevention project "Innovative Lifestyle-Related Disease Prevention Model in the Baltic Sea Region" (www.https:// baltcityprevention.eu/). The project has several aims: 1) to strengthen the institutional capacity of the public health authorities (PHAs) by identifying their needs and requirements in order to better tailor interventions to user-specific groups and 2) to develop an innovative health-related lifestyle intervention model for PHAs. Thus, at first, the survey for PHAs in all participating project countries was planned.

In Latvia, the Institute of Public Health at Riga Stradinš University, the key partner of the BaltCityPrevention Project in collaboration with the Centre of Disease Prevention and Control (CDPC, Rīga) carried out an online survey among health-promoting schools and municipalities. This study aims to explore the experience and needs of health-promoting schools and municipalities in Latvia for implementing health-promotion interventions in order better to understand the context for developing the new intervention model.

\section{Material and methods}

Data were collected from December 2017 to January 2018 by using the online questionnaire, which was developed by the International Partners' Group of the BaltCityPrevention project.

The standardised questionnaire was developed with the aim of collecting information on the experience of developing and implementing health promotion interventions for public 
health authorities by covering the major issues: 1) major target groups for health promotion interventions; 2) tools used for situational assessment used for health promotion intervention planning; 3) major target areas; 4) main partners for health promotion interventions; 5) barriers and difficulties in planning and implementing health promotion interventions; and 6) needs in developing and implementing health promotion interventions.

The online questionnaire was anonymous, and the link for accessing the questionnairewas sent to all 100 municipal schools (91 schools and 9 pre-schools) and 112 municipalities registered in the National Health Promoting Networks by the CDPC representatives using Google Forms. One reminder was sent to all participants after two weeks. The response rate was $82 \%(n=82)$ and $38 \%(n=42)$ for schools and municipalities, respectively. A total of $76.8 \%$ of the responding schools and $92.9 \%$ of municipalities reported having experience in developing health-promotion interventions. Thus, only schools $(n=63)$ and municipalities $(n=39)$ with previous experience in health promotion intervention development were included in the data analysis for this study.

Descriptive and non-parametric statistics were used to calculate percentages with $95 \%$ confidence intervals for each of the response given and to examine the differences between health-promoting schools and municipalities. The statistical software IBM SPSS 22.0 was used for statistical analysis.

\section{Results}

In Table 1, the characteristics of the experiences in implementing health promotion interventions at health-promoting schools and municipalities in Latvia are summarised.

The main target groups for health interventions for schools were school-aged children and parents (79.4\% and 58.7\%, respectively), but for municipalities, the targets were schoolaged children and seniors (76.9\% and $74.4 \%$, respectively). Adolescents, as a particular target group in health promotion interventions, were frequently reported for both schools and municipalities $(58.7 \%$ and $61.5 \%$, respectively).

Most $(90.5 \%)$ of the studied schools $(n=57)$ and $82.1 \%(n=32)$ of municipalities used specific methods for the situational assessment within the planning process of health promotion interventions. Of those having had the experience in conducting the situational assessment, the surveys using questionnaires, target groups or community meetings, and desk research (secondary data analysis) were significantly $(p<0.05)$ more frequently reported tools for the situational assessments for both schools and municipalities.

The primary general focus of health-promotion interventions for both schools and municipalities concerned health behaviour changes. For schools, physical activity, nutrition (81.0\%), also injuries $(69.8 \%)$ and violence $(46.0 \%)$ were the most common focus areas for health-promotion interventions. In comparison with other specific target areas for health promotion interventions, the municipalities significantly and more frequently reported interventions on physical activity $(94.9 \%)$ and nutrition $(76.9 \%)(p<0.001)$ followed by mental health and sexual health and family planning.

Health professionals (58.7\%) and local municipalities $(54.0 \%)$ were the principal collaborative partners for health interventions followed by non-governmental organisations $(36.5 \%)$ and other schools or educational institutions $(31.7 \%)$ within the schools. The municipalities mainly reported collaborations with schools or other educational institutions (89.7\%) and non-governmental organisations (76.9\%). Partnerships with target group representatives and non-governmental organisations in health-promotion interventions were significantly more prevalent for municipalities than schools $(p<0.001)$. As the collaborative 
Table 1. Key characteristics of experience in health promotion interventions at health promotion schools and municipalities in Latvia $(\%)^{\mathrm{a}}$.

\begin{tabular}{|c|c|c|}
\hline Studied areas & $\begin{array}{c}\text { Health-Promoting } \\
\text { Schools, } \\
\%[95 \% \mathrm{CI}]\end{array}$ & $\begin{array}{c}\text { Health-Promoting } \\
\text { Municipalities, } \\
\%[95 \% \mathrm{CI}]\end{array}$ \\
\hline \multicolumn{3}{|c|}{ Target groups for health promotion interventions } \\
\hline School-aged children & $79.4[67.8,87.5]$ & $76.9[61.7,87.3]$ \\
\hline Pre-school children & $36.5[25.7,48.9]$ & $53.8[38.6,68.4]$ \\
\hline Adolescents & $58.7[46.4,70.0]$ & $61.5[45.9,75.1]$ \\
\hline Parents & $58.7[46.4,70.0]$ & $48.7[33.9,63.8]$ \\
\hline Adults & $14.3[7.7,25.0]$ & $66.7[51.0,79.4]$ \\
\hline Seniors & $1.6[2.8,8.5]$ & $74.4[59.0 ; 85.4]$ \\
\hline Persons with special health needs & $6.3[2.5,15.2]$ & $33.3[20.6,49.0]$ \\
\hline General population & $1.6[2.8,8.5]$ & $59.0[43.4,72.3]$ \\
\hline \multicolumn{3}{|l|}{ Tools used for situational assessment } \\
\hline Interviews & $12.5[5.0,28.1]$ & $19.3[11.1,31.3]$ \\
\hline Questionnaires & $66.7[53.7,77.5]$ & $59.4[42.3,74.5]$ \\
\hline Desk research (secondary data analysis) & $42.1[30.2,55.0]$ & $31.3[18.0,48.6]$ \\
\hline Target group/community meetings & $77.2[65.0,88.2]$ & $62.5[45.2,77.1]$ \\
\hline \multicolumn{3}{|c|}{ Target areas for health promotion interventions } \\
\hline \multicolumn{3}{|l|}{ General } \\
\hline Health behaviour change & $87.3[76.9,93.4]$ & $89.7[76.4,95.9]$ \\
\hline Disease prevention & $57.1[44.9,68.6]$ & $71.8[56.2,83.5]$ \\
\hline \multicolumn{3}{|l|}{ Specific } \\
\hline Injuries & $69.8[57.6,79.8]$ & $10.3[4.1,23.5]$ \\
\hline Violence & $46.0[34.3,58.2]$ & $23.1[12.6,38.3]$ \\
\hline Mental health & $27.0[17.6,39.0]$ & $35.9[22.7,51.6]$ \\
\hline Physical activity & $81.0[69.6,88.7]$ & $94.9[83.1,98.6]$ \\
\hline Nutrition & $81.0[69.6,88.7]$ & $76.9[61.7,87.3]$ \\
\hline Sleep & $20.6[12.5,32.2]$ & $0.0[0.0,9.0]$ \\
\hline Sexual health \& family planning & $14.3[7.7,25.0]$ & $35.9[22.7,51.6]$ \\
\hline \multicolumn{3}{|c|}{ Collaborative partners for health promotion interventions } \\
\hline Health professionals & $58.7[46.4,70.0]$ & $64.1[48.4,77.3]$ \\
\hline Schools/educational institutions*** & $31.7[21.6,44.0]$ & $89.7[76.4,95.9]$ \\
\hline Non-governmental organizations $* * *$ & $36.5[25.7,48.8]$ & $76.9[61.7,87.3]$ \\
\hline Local municipalities & $54.0[41.8,65.7]$ & $51.3[36.2,66.1]$ \\
\hline Target group representatives $* * *$ & $23.8[15.0,35.6]$ & $59.0[43.4,72.9]$ \\
\hline Public health institutions & $34.9[24.3,47.2]$ & $23.1[12.6,38.3]$ \\
\hline Mass media & $19.0[11.2,30.4]$ & $35.9[22.7,51.6]$ \\
\hline Small and medium enterprises* & $7.9[3.4,17.3]$ & $23.1[12.6,38.3]$ \\
\hline
\end{tabular}


Table 2. Experienced difficulties and expected needs in implementing health promotion interventions at health promoting schools and municipalities in Latvia $(\%)^{\mathrm{a}}$.

\begin{tabular}{|c|c|c|}
\hline Studied areas & $\begin{array}{c}\text { Health-Promoting } \\
\text { Schools, } \\
\%[95 \% \mathrm{CI}]\end{array}$ & $\begin{array}{c}\text { Health-Promoting } \\
\text { Municipalities, } \\
\%[95 \% \text { CI }]\end{array}$ \\
\hline \multicolumn{3}{|c|}{ Difficulties in planning and implementing health promotion interventions } \\
\hline \multicolumn{3}{|l|}{ Resources } \\
\hline Lack of funding** & $63.5[46.4,70.0]$ & $35.9[22.7,51.6]$ \\
\hline Limited access to expertise and professionals & $30.2[20.2,42.4]$ & $48.7[33.9,63.8]$ \\
\hline Lack of e-tools & $12.8[5.6,26.7]$ & $19.0[11.2,30.4]$ \\
\hline Lack of specific health data*** & $11.1[5.5,21.2]$ & $38.5[24.9,54.1]$ \\
\hline Limited access to evidence-based research & $15.4[7.2,29.7]$ & $6.3[2.5,15.2]$ \\
\hline \multicolumn{3}{|c|}{ Skills and competencies needed for health promotion interventions development } \\
\hline $\begin{array}{l}\text { Lack of knowledge about how to approach the target } \\
\text { group** }\end{array}$ & $28.6[18.9,40.1]$ & $53.8[38.6,68.4]$ \\
\hline $\begin{array}{l}\text { Lack of knowledge about the effectiveness of health } \\
\text { behaviour change interventions }\end{array}$ & $9.5[4.4,19.3]$ & $20.5[10.8,35.5]$ \\
\hline $\begin{array}{l}\text { Lack of competence to understand and translate } \\
\text { research }\end{array}$ & $3.2[0.9,10.9]$ & $5.1[1.4,16.9]$ \\
\hline Lack of intervention evaluation competencies & $4.8[1.6,13.1]$ & $5.1[1.4,16.9]$ \\
\hline \multicolumn{3}{|l|}{ Leadership and partnership } \\
\hline Lack of cooperation between public health authorities & $27.0[17.6,39.0]$ & $38.5[24.9,54.1]$ \\
\hline Lack of inter-sectoral collaboration*** & $1.6[0.3,8.5]$ & $20.5[10.8,35.5]$ \\
\hline Limited autonomy to make decisions & $6.3[2.5,15.2]$ & $17.9[9.0,32.7]$ \\
\hline \multicolumn{3}{|c|}{ Needs in developing and implementing health promotion interventions } \\
\hline \multicolumn{3}{|l|}{ Resources } \\
\hline Financial resources & $74.6[62.7,83.7]$ & $71.8[56.2,83.5]$ \\
\hline Health needs assessment** & $30.2[20.2,42.4]$ & $56.4[40.1,70.7]$ \\
\hline Expert opinions & $30.2[20.2,42.4]$ & $33.3[20.6,49.0]$ \\
\hline E-tools & $34.9[24.3,47.2]$ & $28.2[16.5,43.8]$ \\
\hline \multicolumn{3}{|c|}{ Skills and competencies needed for health promotion interventions development } \\
\hline Sharing of best practices & $69.8[57.6,79.8]$ & $69.2[53.6,81.4]$ \\
\hline $\begin{array}{l}\text { Increased knowledge about the effectiveness of health } \\
\text { promotion interventions }\end{array}$ & $28.6[18.9,40.1]$ & $30.1[18.6,46.4]$ \\
\hline \multicolumn{3}{|l|}{ Leadership and partnership } \\
\hline Political support*** & $4.8[1.6,13.1]$ & $38.4[24.9,54.1]$ \\
\hline Administrative support* & $22.2[13.7,33.9]$ & $43.6[29.3,59.0]$ \\
\hline $\begin{array}{l}\text { Stronger collaboration with small and medium } \\
\text { enterprises }\end{array}$ & $39.7[28.5,52.0]$ & $25.6[14.6,41.1]$ \\
\hline
\end{tabular}

partners for health promotion institutions, small/medium enterprises and universities were the least likely to be reported by both schools and municipalities.

When studying the previous difficulties in implementing health promotion interventions, the schools reported lack of funding (63.5\%), access to expertise $(30.2 \%)$, and the lack of knowledge about the methods for approaching different target groups (28.6\%) as the 
main barriers for effective health promotion (Table 2). Twenty-seven percent of schools also reported previous difficulties in cooperating with public health authorities for healthpromotion interventions.

Among the studied municipalities, the most common obstacles when implementing health interventions were the lack of knowledge aimed at reaching the target groups (53.8\%) and limited access to expertise and professionals in the field $(48.7 \%)$ followed by the lack of funding (35.9\%), lack of specific health data (38.5\%), and difficulties in cooperation among the public health authorities $(38.5 \%)$. Some $(20.5 \%)$ of the studied municipalities also reported lack of knowledge of the effectiveness of health behaviour change interventions and the lack of inter-section collaboration.

The proportion of those studied schools and municipalities that reported difficulties in translating evidence-based knowledge and evaluating the interventional outcomes was relatively low.

More than $70 \%$ of both schools and municipalities reported the for more health-promotion intervention funding. However, also sharing best practices used to be an important issue in almost $70 \%$ of the studied participants. The requirement for health needs assessment was more frequently reported among municipalities (56.4\%) than schools (30.2\%), and the need for better political and administrative support for developing health-promotion interventions was more relevant for municipalities than for schools.

\section{Conclusions}

To our knowledge, this is the first published study showing some of the key characteristics of the previous practices, difficulties, and needs in planning and implementing health promotion interventions of those schools and municipalities belonging to the Health Promotion Networks in Latvia.

In general, for both school and municipalities, the school-aged children were the most common target group in health-promotion interventions. As all of the studied schools were municipal schools, some mixing and overlapping may have occurred between the interventions implemented primarily by schools or municipalities. Parents and adolescents also were frequently reported to be target groups at the school-level health promotion interventions. Among the studied municipalities, the health promotion interventions were also often targeted to seniors and the general adult population. The smallest proportion of municipalities reported persons with special health needs as a special target group for health promotion interventions. Physical activity and nutrition were by far the most frequently reported specific target areas in previous interventions of health behaviour changes for both schools and municipalities.

The spectrum of collaborative partners for health promotion interventions is different for schools and municipalities, which may reflect the existing true differences in governance and leadership between schools and municipalities. The municipalities identified schools or other educational institutions as key partners for collaboration in health promotion the most. The proportion of schools reporting local municipalities and health professionals as the main collaborative partners for health promotion was the highest, and there were no significant differences between the two groups. Both schools and municipalities reported less frequent collaboration with small and medium enterprises. Some existing administrative barriers and legal aspects might explain this; however, these findings suggest that for building multi-section partnerships, focusing on closer collaboration with the private sector could be considered. 
When studying previous difficulties and barriers in implementing health promotion interventions, lack of funding was significantly less frequently reported by health-promoting municipalities (36\%) in comparison with schools $(63 \%)$. There are fundamental differences in fund management and administration systems at municipal and school-levels. However, the relatively low percentage of those health promoting municipalities reporting the lack funding for health promotion might be also explained by more opportunities to receive funding for health promotion at the local level for municipalities in Latvia in the frame of the European Social Fund project "Measures for Health Promotion and Disease Prevention at Local Level" lasting from 2016 to 2022 .

The proportion of those schools reporting collaborations with the target group in planning or implementing health promotion interventions was relatively low(24\%), and only $29 \%$ of schools reported the lack of knowledge about how to approach the target group as the main challenge in implementing health-promotion interventions in the past. In comparison, almost $59 \%$ of the studied municipalities reported previous collaboration with the target groups' representatives in health-promotion when more than half acknowledged the lack of knowledge in better target group approach. These findings might suggest that the use of a participatory approach when the target group is being involved in the different phases of health promotion interventions should be much better promoted and supported.

This study provides an overview of experience in implementing health promotion interventions at health promoting schools and municipalities in Latvia. The findings of this study could be used for training and support system planning in order to increase the capacity for better health promotion. More focused and detailed studies might be important for increasing the awareness of both specific features and the larger context of developing health promotion interventions in local settings.

The authors are grateful to all study participants within the National Health Promoting Networks in Latvia for their response. The team of the BaltCityPrevention project from the Institute of Public Health at Riga Stradinš University thanks the members from the Department of Health Promotion at the Centre of Disease Prevention and Control for their support and collaboration during data collection process.

\section{References}

[1] WHO, The Ottawa Charter for Health Promotion (1986). Available at: https:// wWw. who.int/healthpromotion/conferences/previous/ottawa/en/ [viewed 2019. 21.01]

[2] M. Dobbins, H. Husson, K. DeCorby, R.L. LaRocca, School-Based Physical Activity Programs for Promoting Physical Activity and Fitness in Children and Adolescents Aged 6 to 18, Cochrane Database Syst. Rev., Cd007651 (2013)

[3] C.E. Evans, M.S. Christian, C.L. Cleghorn, D.C. Greenwood, J.E. Cade, Systematic Review and Meta-Analysis of School-Based Interventions to Improve Daily Fruit and Vegetable Intake in Children Aged 5 to 12 Y, Am. J. Clin. Nutr. 96, 889 (2012)

[4] C. Knai, J. Pomerleau, K. Lock, M. McKee, Getting Children to Eat More Fruit and Vegetables: A Systematic Review, Prev. Med. 42, 85 (2006)

[5] R.E. Thomas, J. McLellan, R. Perera, School-Based Programmes for Preventing Smoking, Cochrane Database Syst. Rev., Cd001293 (2013)

[6] R. Langford, C. Bonell, K. Komro, S. Murphy, D. Magnus, E. Waters, L. Gibbs, R. Campbell, The Health Promoting Schools Framework: Known Unknowns and an Agenda for Future Research, Health Educ. Behav. 44, 463 (2017) 
[7] WHO, Global school health initiative (1995). Available at: http://www.who.int/ school_youth_health/gshi/en/ [viewed 10.01.2019]

[8] M.K. Helgesen, E. Fosse, S. Hagen, Capacity to Reduce Inequities in Health in Norwegian Municipalities, Scand. J. Public Health 45, 77 (2017)

[9] N. Simonsen-Rehn, R. Laamanen, M. Brommels, S. Suominen, Determinants of Effective Health Promotion Actions in Local Contexts: A Study of the Perceptions of Municipal Politicians, Int. J. Public Health 57, 787 (2012)

[10] E.V. Jansson, P.E. Tillgren, Health Promotion at Local Level: A Case Study of Content, Organization, and Development in Four Swedish Municipalities, BMC Public Health 10, 455 (2010)

[11] S. Michie, M.M. van Stralen, R. West, The Behaviour Change Wheel: A New Method for Characterising and Designing Behaviour Change Interventions, Implement Sci. 6, $42(2011)$

[12] L.K. Bartholomew, C.M. Markham, R.A.C. Ruiter, M.E. Fernàndez, G. Kok, G.S. Parcel, Planning health promotion programs: An intervention mapping approach (4th ed.) (Hoboken, NJ: Wiley, 2016)

[13] L. Green, M.K. Kreuter, Health program planning an educational and ecological approach. (4th Ed.) (New York: McGraw Hill, 2005) 